\title{
STABILITY OF FINITE DIFFERENCE SCHEMES FOR COMPLEX DIFFUSION PROCESSES
}

\author{
ADÉRITO ARAÚJO *, SílVIA BARBEIRO †, AND PEDRO SERRANHO ‡
}

Key words. finite differences, nonlinear complex diffusion, stability

AMS subject classifications. 65M12, 65M06

\begin{abstract}
In this paper we present a rigorous proof for the stability of a class of finite difference schemes applied to nonlinear complex diffusion equations. Complex diffusion is a common and broadly used denoising procedure in image processing. To illustrate the theoretical results we present some numerical examples based on an explicit scheme applied to a nonlinear equation in context of image denoising.
\end{abstract}

1. Introduction. The main result of this paper is the proof of a stability condition for a class of finite difference schemes for nonlinear complex diffusion. The stability condition for the linear case is very well known and widely documented in literature $[12,13]$. In [3] the authors derive, under suitable conditions, a stability result for the linear complex case. Our result, on top of being a non-trivial generalization of the stability condition for the nonlinear case, also requires less regularity in the diffusion coefficient than [3].

Diffusion processes are commonly used in image processing, as for example in inpainting [8], stereo vision [16] or optical flow [2]. Another important application of diffusion processes in image processing is noise removal $[1,11,10,14,15]$. The main idea is that if one pixel is affected by noise, than the noise should be diffused among the neighboring pixels in order to smooth the region. In this way proper diffusion partial differential equations have been considered to achieve this end. Taking the nonlinear diffusion equation

$$
\frac{\partial u(x, t)}{\partial t}=\operatorname{div}(D(x, t, u) \nabla u(x, t))
$$

where $u(x, t)$ represents the denoised image at time $t$ with the initial noisy image $u(x, 0)$, the choice of the diffusion parameter $D$ plays a very important role for the purpose of denoising. Here $\nabla$ and div denote the gradient and the divergence operators, respectively. Roughly speaking, one wants $D$ to allow diffusion on homogeneous areas affected only by noise and to forbid diffusion on edges to preserve features of the original denoised image. In this way, several expressions for $D$ have been suggested. The first approaches indicated that $D$ should depend on the gradient of $u$ with an inverse proportion $[10,14,15]$. However, this kind of approach has some handicaps. For instance, within a ramp edge the diffusion coefficient is similar along all the edge delaying the diffusion process, not distinguishing between the end points and interior points of the ramp edge where diffusion should differ. Therefore, the use of the Laplacian in the definition of $D$ was suggested as being more appropriate since

${ }^{*}$ CMUC, Department of Mathematics, University of Coimbra, Apartado 3008, EC Universidade, 3001-454 Coimbra, Portugal(alma@mat.uc.pt).

${ }^{\dagger}$ CMUC, Department of Mathematics, University of Coimbra, Apartado 3008, EC Universidade, 3001-454 Coimbra, Portugal(silvia@mat.uc.pt).

${ }^{\ddagger}$ Mathematics Section, Department of Science and Technology, Open University, Campus TagusPark - Av. Dr. Jacques Delors, 2740-122 Porto Salvo, Oeiras, Portugal and IBILI, Faculty of Medicine, University of Coimbra, Portugal (pserranho@univ-ab.pt). 
it has a higher amplitude near the end points and lower magnitude elsewhere, namely by considering

$$
D=\frac{1}{1+|\Delta u|^{2}}
$$

The drawback is that the computation of a higher order derivative is needed leading to higher ill-posedness of the finite difference scheme in the first steps while the image is strongly affected by noise. To overcome this problem, Gilboa et al. [7] suggested to use complex diffusion. The use of a complex diffusion coefficient turns the partial differential equation into some sort of combination between the heat and the Schrödinger equation $[4,5]$. Having the goal of preserving image features in view, Gilboa et al. [7] suggested

$$
D=\frac{e^{i \vartheta}}{1+\left(\frac{\operatorname{Im}(u)}{\kappa \vartheta}\right)^{2}},
$$

with $\vartheta \approx 0$ and $\kappa>0$ a normalization constant, involving an approximation to the Laplacian of the image, namely by using the identity

$$
\lim _{\vartheta \rightarrow 0} \frac{\operatorname{Im}(u(., t))}{t \vartheta}=G * \Delta u(., 0)
$$

where $G$ is a Gaussian and therefore the convolution with $G$ represents a low pass filter. We note that the right hand side is a low-pass filter of the structure of edges of the initial image $u(., 0)$ given by its Laplacian, and therefore the choice of $D$ as in (1.3) penalizes diffusion across edges.

Complex diffusion proved to be much better conditioned numerically and successfully applied in medical imaging despeckling and denoising $[6,11]$. More recently an improvement of $D$ by making $\kappa$ adaptive was also strongly suggested [1]. Having in mind that explicit finite difference schemes for the discretization of the equation are usually considered in order to obtain fast computational methods, it is important to look for a stability condition for those schemes. It is known that for a real positive diffusion parameter $D$ in the one-dimensional case, the classical explicit first order in time and second order in space method is stable if

$$
\Delta t \leq \frac{h^{2}}{2 \max D}
$$

where $\Delta t$ and $h$ are, respectively, the temporal and spatial discretization steps. This upper bound has generally been used by engineers when implementing complex diffusion, though no rigorous proof of stability condition was presented in this case. For the case where $D$ is complex, not dependent on $u$ and sufficiently smooth $\left(D(\cdot, t) \in C^{3}(\Omega)\right)$, Tony Chan and Longjun Shen, in [3], prove that if

$$
\Delta t \leq \frac{h^{2}}{2 \max \frac{|D|^{2}}{D_{R}}} .
$$

where $D_{R} \neq 0$ is the real part of $D$, the classical explicit first order in time and second order in space method is stable. However, to the best of our knowledge there is no rigorous proof to the stability condition for the complex nonlinear case. 
The paper is organized as follows. In Section 2 the complex diffusion equation is presented and the details on the considered class of numerical schemes are also given. Next, the stability condition is proven. We only present in detail the proof for the unidimensional case and Neumann boundary conditions. Remark 1 is devoted to the generalization of the proof for the easier Dirichlet case. We present numerical examples in Section 3 to illustrate the stability condition derived in Section 2. We end the paper with a section dedicated to conclusions and possible future work.

2. Complex diffusion equation and stability of the numerical scheme. Let $\Omega \subset \mathbb{R}^{d}, d \geq 1$, be is the cartesian product of open intervals in $\mathbb{R}$, with boundary $\Gamma=\partial \Omega$,

$$
\left.\Omega=\prod_{j=1}^{d}\right] a_{j}, b_{j}[,
$$

with $a_{j}, b_{j} \in \mathbb{R}$. Let $\left.\left.Q=\Omega \times\right] 0, T\right]$, with $T>0$, and $u: \bar{Q}=\bar{\Omega} \times[0, T] \longrightarrow \mathbb{C}$. We consider a nonlinear diffusion process with a non-constant complex coefficient $D(x, t, u)=$ $D_{R}(x, t, u)+i D_{I}(x, t, u)$, where $D_{R}(x, t, u)$ and $D_{I}(x, t, u)$ are real functions. We also need to assume that

$$
D_{R}(x, t, u) \geq 0, \quad(x, t) \in \bar{Q},
$$

and that there exists a constant $L>0$ such that

$$
0<|D(x, t, u)| \leq L, \quad(x, t) \in \bar{Q} .
$$

We define the initial boundary value problem for the unknown function $u$

$$
\left\{\begin{array}{l}
\frac{\partial u}{\partial t}(x, t)=\operatorname{div}(D(x, t, u) \nabla u(x, t)), \quad(x, t) \in Q \\
u(x, 0)=u^{0}(x), \quad x \in \bar{\Omega}, \\
\alpha u(x, t)+\beta \frac{\partial u}{\partial \nu}(x, t)=0, \quad x \in \Gamma, \quad t \in[0, T],
\end{array}\right.
$$

where $\frac{\partial u}{\partial \nu}$ denotes the derivative in the direction of the exterior normal $\nu$ to $\Gamma$. More details, namely with $D$ defined by (1.3), can be found in [7].

For the boundary conditions we consider that

$$
\alpha \beta=0 \quad \text { and } \quad \alpha+\beta \neq 0 .
$$

Let us construct a mesh on $\bar{Q}$. For the temporal interval we consider the mesh

$$
0=t^{0}<t^{1}<\cdots<t^{M-1}<t^{M}=T
$$

where $M \geq 1$ is an integer and $t^{m+1}-t^{m}=\Delta t^{m}, m=0, \ldots, M-1$. Let $h_{k}$ denote the mesh-size in the $k$ th spatial coordinate direction, such that $h_{k}=\left(b_{k}-a_{k}\right) / N_{k}$, for $k=1, \ldots, d$, and $N_{k} \geq 2$ an integer. Let $h=\max h_{k}$ and $\Delta t=\max \Delta t^{m}$. The set of the points

$$
x_{j}=\left(a_{1}+j_{1} h_{1}, \ldots, a_{d}+j_{d} h_{d}\right), 0 \leq j_{k} \leq N_{k}, k=1, \ldots, d
$$


defines a space grid that we denote by $\bar{\Omega}_{h}$. We associate the point $\left(x_{j}, t^{m}\right)$ with the coordinate $(j, m)=\left(j_{1}, \ldots, j_{d}, m\right)$.

We define a mesh in $\bar{Q}$, which we denote by $\bar{Q}_{h}^{\Delta t}$, by the cartesian product of the space grid $\bar{\Omega}_{h}$ and a grid in the temporal domain. Let $Q_{h}^{\Delta t}=\bar{Q}_{h}^{\Delta t} \cap Q$ and $\Gamma_{h}^{\Delta t}=\bar{Q}_{h}^{\Delta t} \cap \Gamma \times[0, T]$.

We denote by $V_{j}^{m}$ the value of a mesh function $V$, defined on $\bar{Q}_{h}^{\Delta t}$, at the point $\left(x_{j}, t^{m}\right)$. We define the forward and backward finite differences with respect to $\left(x_{j}, t^{m}\right)$ in the $k$ th spatial direction by

$$
\delta_{k}^{+} V_{j}^{m}=\frac{V_{j+e_{k}}^{m}-V_{j}^{m}}{h_{k}}, \quad \delta_{k}^{-} V_{j}^{m}=\frac{V_{j}^{m}-V_{j-e_{k}}^{m}}{h_{k}},
$$

where $e_{k}$ denotes the $k$ th element of the natural basis in $\mathbb{R}^{d}$.

On $\bar{Q}_{h}^{\Delta t}$ we approximate (2.4) by the one-parameter family of finite difference schemes

$$
\left\{\begin{array}{l}
\frac{U_{j}^{m+1}-U_{j}^{m}}{\Delta t^{m}}=\sum_{k=1}^{d} \delta_{k}^{+}\left(D_{j-(1 / 2) e_{k}}^{m+\theta} \delta_{k}^{-} U_{j}^{m+\theta}\right) \text { in } \tilde{Q}_{h}^{\Delta t}, \\
U_{j}^{0}=u_{0}\left(x_{j}\right) \text { in } \bar{\Omega}_{h}, \\
\alpha U_{j}^{m}+\frac{\beta}{2} \sum_{k=1}^{d}\left(\delta_{k}^{+} U_{j}^{m}+\delta_{k}^{-} U_{j}^{m}\right) \cdot \nu_{k}=0 \quad \text { in } \Gamma_{h}^{\Delta t},
\end{array}\right.
$$

where $V_{j}^{m+\theta}=\theta V_{j}^{m+1}+(1-\theta) V_{j}^{m}, \theta \in[0,1], U_{j}^{m}$ represents the approximation of $u\left(x_{j}, t^{m}\right)$ and

$$
D_{j-(1 / 2) e_{k}}^{m}=\frac{D\left(x_{j}, t^{m}, U_{j}^{m}\right)+D\left(x_{j-e_{k}}, t^{m}, U_{j-e_{k}}^{m}\right)}{2} .
$$

We use the notation $\tilde{Q}_{h}^{\Delta t}$ for the set $Q_{h}^{\Delta t}$ or $\bar{Q}_{h}^{\Delta t}$, respectively, in the case of Dirichlet or Neumann boundary conditions, and $\nu_{k}$ represents the $k$ th component of the normal vector $\nu$.

If $\theta=0$ then (2.9) is an explicit method. For $\theta>0$ the method is implicit involving a nonlinear system of equations. For computational purposes an efficient strategy to deal with the nonlinearity has to be considered.

Assuming enough regularity for the solution of (2.4), we may easily prove that the method is consistent with the differential problem of order $\left(h^{2}, \Delta t\right)$ if $\theta \neq \frac{1}{2}$, and $\left(h^{2},(\Delta t)^{2}\right)$ if $\theta=\frac{1}{2}$. Note that, in this last case, the time discretization corresponds to the Crank-Nicolson method.

We will now investigate the stability of the finite difference scheme (2.9). The approach we use here has some analogies with strategies more commonly used in the context of the finite element method but it has already been used in the context of the finite difference method (see e.g. [12]).

TheOrEm 2.1. Let (2.2) and (2.3) hold. If $\theta \in\left[\frac{1}{2}, 1\right]$ then the method (2.9) is unconditionally stable. If $\theta \in\left[0, \frac{1}{2}[\right.$ then the method (2.9) is stable under the condition

$$
\Delta t^{m} \leq \frac{\left(\min \left\{h_{1}, \ldots, h_{d}\right\}\right)^{2}}{2 d(1-2 \theta) \max _{x_{j} \in \bar{\Omega}_{h}} \frac{\left|D_{j}^{m+\theta}\right|^{2}}{D_{R j}^{m+\theta}}}, \quad m=1, \ldots, M-1,
$$


provided that there exists some $\xi$ such that

$$
0<\xi \leq D_{R j}^{m+\theta} \quad \forall j, m .
$$

To prove this result we will consider the unidimensional case and Neumann boundary conditions $(\alpha=0)$. For $d \geq 2$ or Dirichlet boundary conditions the proof follows the same steps.

We rewrite (2.9) as a system by separating the real and imaginary parts, $U_{R}$ and $U_{I}$, respectively, of the main variable $U=\left(U_{0}, \ldots, U_{N}\right)$. We shall then study the stability of the family of finite difference schemes: find $U_{j}^{m} \approx u\left(x_{j}, t^{m}\right), j=0, \ldots, N$, $m=0, \ldots, M$, such that

$$
\left\{\begin{array}{l}
\frac{U_{R j}^{m+1}-U_{R j}^{m}}{\Delta t^{m}}=\delta_{x}^{+}\left(D_{R j^{-}}^{m+\theta} \delta_{x}^{-} U_{R j}^{m+\theta}\right)-\delta_{x}^{+}\left(D_{I j^{-}}^{m+\theta} \delta_{x}^{-} U_{I j}^{m+\theta}\right), \begin{array}{l}
j=0, \ldots, N, \\
m=0, \ldots, M-1,
\end{array} \\
\frac{U_{I j}^{m+1}-U_{I j}^{m}}{\Delta t^{m}}=\delta_{x}^{+}\left(D_{I j^{-}}^{m+\theta} \delta_{x}^{-} U_{R j}^{m+\theta}\right)+\delta_{x}^{+}\left(D_{R j^{-}}^{m+\theta} \delta_{x}^{-} U_{I j}^{m+\theta}\right), \begin{array}{l}
j=0, \ldots, N, \\
m=0, \ldots, M-1,
\end{array} \\
U_{R j}^{0}=u_{R}^{0}\left(x_{j}\right), U_{I j}^{0}=u_{I}^{0}\left(x_{j}\right), \quad j=0, \ldots, N, \\
\delta_{x}^{+} U_{R 0}^{m}+\delta_{x}^{-} U_{R 0}^{m}=0, \delta_{x}^{+} U_{R N}^{m}+\delta_{x}^{-} U_{R N}^{m}=0, \quad m=0, \ldots, M, \\
\delta_{x}^{+} U_{I 0}^{m}+\delta_{x}^{-} U_{I 0}^{m}=0, \delta_{x}^{+} U_{I N}^{m}+\delta_{x}^{-} U_{I N}^{m}=0, \quad m=0, \ldots, M,
\end{array}\right.
$$

where

$$
D_{j^{-}}^{m}=\frac{D\left(x_{j-1}, t^{m}, U_{j-1}^{m}\right)+D\left(x_{j}, t^{m}, U_{j}^{m}\right)}{2}, j=1, \ldots, N, m=0, \ldots, M .
$$

In (2.13) we need the extra points $x_{-1}=x_{0}-h$ and $x_{N+1}=x_{N}+h$ and we define $D_{0^{-}}^{m}=D_{1^{-}}^{m}, D_{(N+1)^{-}}^{m}=D_{N^{-}}^{m}$.

We consider the discrete $L^{2}$ inner products

$$
(U, V)_{h}=\frac{h}{2} U_{0} \bar{V}_{0}+\sum_{j=1}^{N-1} h U_{j} \bar{V}_{j}+\frac{h}{2} U_{N} \bar{V}_{N}
$$

and

$$
(U, V)_{h^{*}}=\sum_{j=1}^{N} h U_{j} \bar{V}_{j},
$$

and their corresponding norms

$$
\|U\|_{h}=(U, U)_{h}^{1 / 2} \text { and }\|U\|_{h^{*}}=(U, U)_{h^{*}}^{1 / 2} .
$$

Multiplying both members of the first and second equations of (2.13) by, respectively, $U_{R}^{m+\theta}$ and $U_{I}^{m+\theta}$, according to the discrete inner product $(\cdot, \cdot)_{h}$ and using summation by parts we obtain

$$
\left(\frac{U_{R}^{m+1}-U_{R}^{m}}{\Delta t^{m}}, U_{R}^{m+\theta}\right)_{h}+\left\|\left(D_{R^{-}}^{m+\theta}\right)^{1 / 2} \delta_{x}^{-} U_{R}^{m+\theta}\right\|_{h^{*}}^{2}=\left(D_{I^{-}}^{m+\theta} \delta_{x}^{-} U_{I}^{m+\theta}, \delta_{x}^{-} U_{R}^{m+\theta}\right)_{h^{*}}
$$


and

$$
\left(\frac{U_{I}^{m+1}-U_{I}^{m}}{\Delta t^{m}}, U_{I}^{m+\theta}\right)_{h}+\left\|\left(D_{R^{-}}^{m+\theta}\right)^{1 / 2} \delta_{x}^{-} U_{I}^{m+\theta}\right\|_{h^{*}}^{2}=-\left(D_{I^{-}}^{m+\theta} \delta_{x}^{-} U_{R}^{m+\theta}, \delta_{x}^{-} U_{I}^{m+\theta}\right)_{h^{*}} .
$$

Then

$$
\left(\frac{U^{m+1}-U^{m}}{\Delta t^{m}}, U^{m+\theta}\right)_{h}+\left\|\left(D_{R^{-}}^{m+\theta}\right)^{1 / 2} \delta_{x}^{-} U^{m+\theta}\right\|_{h^{*}}^{2}=0
$$

Since we can write

$$
U^{m+\theta}=\Delta t^{m}\left(\theta-\frac{1}{2}\right) \frac{U^{m+1}-U^{m}}{\Delta t^{m}}+\frac{U^{m+1}+U^{m}}{2},
$$

we obtain

$\Delta t^{m}\left(\theta-\frac{1}{2}\right)\left\|\frac{U^{m+1}-U^{m}}{\Delta t^{m}}\right\|_{h}^{2}+\frac{\left\|U^{m+1}\right\|_{h}^{2}-\left\|U^{m}\right\|_{h}^{2}}{2 \Delta t^{m}}+\left\|\left(D_{R j^{-}}^{m+\theta}\right)^{1 / 2} \delta_{x}^{-} U^{m+\theta}\right\|_{h^{*}}^{2}=0$.

Let us now consider two different cases. First, we suppose that $\theta \in\left[\frac{1}{2}, 1\right]$. In this case $\theta-\frac{1}{2} \geq 0$ and from (2.22) we immediately obtain that

$$
\left\|U^{m+1}\right\|_{h} \leq\left\|U^{m}\right\|_{h}, \quad m=0, \ldots, M-1,
$$

which proves that the scheme is stable without any limitation in the time step.

Now, let us consider that $\theta \in\left[0, \frac{1}{2}[\right.$. In this case,

$$
\frac{\left\|U^{m+1}\right\|_{h}^{2}-\left\|U^{m}\right\|_{h}^{2}}{2 \Delta t^{m}}+\left\|\left(D_{R^{-}}^{m+\theta}\right)^{1 / 2} \delta_{x}^{-} U^{m+\theta}\right\|_{h^{*}}^{2}=\Delta t^{m}\left(\frac{1}{2}-\theta\right)\left\|\frac{U^{m+1}-U^{m}}{\Delta t^{m}}\right\|_{h}^{2} .
$$

We recall that

$$
\left\|\frac{U^{m+1}-U^{m}}{\Delta t^{m}}\right\|_{h}^{2}=\left\|\frac{U_{R}^{m+1}-U_{R}^{m}}{\Delta t^{m}}\right\|_{h}^{2}+\left\|\frac{U_{I}^{m+1}-U_{I}^{m}}{\Delta t^{m}}\right\|_{h}^{2} .
$$

From the first equation of (2.13) we get

$$
\begin{aligned}
\frac{U_{R j}^{m+1}-U_{R j}^{m}}{\Delta t^{m}}= & \frac{1}{h}\left(D_{R j+1^{-}}^{m+\theta} \delta_{x}^{-} U_{R j+1}^{m+\theta}-D_{R j+1^{-}}^{m+\theta} \delta_{x}^{-} U_{R j+1}^{m+\theta}\right) \\
& -\frac{1}{h}\left(D_{I j+1^{-}}^{m+\theta} \delta_{x}^{-} U_{I j+1}^{m+\theta}-D_{I j+1^{-}}^{m+\theta} \delta_{x}^{-} U_{I j+1}^{m+\theta}\right) .
\end{aligned}
$$

We now consider the norm $\|.\|_{h}$ of both members of (2.26). Using the boundary conditions in (2.13) and applying the inequality $(a-b)^{2} \leq 2 a^{2}+2 b^{2}$, we obtain

$$
\begin{aligned}
\left\|\frac{U_{R}^{m+1}-U_{R}^{m}}{\Delta t^{m}}\right\|_{h}^{2} \leq & \frac{4}{h^{2}}\left\|D_{R^{-}}^{m+\theta} \delta_{x}^{-} U_{R}^{m+\theta}-D_{I^{-}}^{m+\theta} \delta_{x}^{-} U_{I}^{m+\theta}\right\|_{h^{*}}^{2} \\
= & \frac{4}{h^{2}}\left(\left\|D_{R^{-}}^{m+\theta} \delta_{x}^{-} U_{R}^{m+\theta}\right\|_{h^{*}}^{2}+\left\|D_{I^{-}}^{m+\theta} \delta_{x}^{-} U_{I}^{m+\theta}\right\|_{h^{*}}^{2}\right) \\
& -\frac{8}{h} \sum_{j=1}^{N} D_{R j^{-}}^{m+\theta} \delta_{x}^{-} U_{R j}^{m+\theta} D_{I j^{-}}^{m+\theta} \delta_{x}^{-} U_{I j}^{m+\theta} .
\end{aligned}
$$


Likewise, from the second equation of (2.13) we get

$$
\begin{aligned}
\frac{U_{I j}^{m+1}-U_{I j}^{m}}{\Delta t^{m}}= & \frac{1}{h}\left(D_{I j+1^{-}}^{m+\theta} \delta_{x}^{-} U_{R j+1}^{m+\theta}-D_{I j+1^{-}}^{m+\theta} \delta_{x}^{-} U_{R j+1}^{m+\theta}\right) \\
& +\frac{1}{h}\left(D_{R j+1^{-}}^{m+\theta} \delta_{x}^{-} U_{I j+1}^{m+\theta}-D_{R j+1^{-}}^{m+\theta} \delta_{x}^{-} U_{I j+1}^{m+\theta}\right)
\end{aligned}
$$

and then,

$$
\begin{aligned}
\left\|\frac{U_{I}^{m+1}-U_{I}^{m}}{\Delta t^{m}}\right\|_{h}^{2} \leq & \frac{4}{h^{2}}\left\|D_{I^{-}}^{m+\theta} \delta_{x}^{-} U_{R}^{m+\theta}+D_{R^{-}}^{m+\theta} \delta_{x}^{-} U_{I}^{m+\theta}\right\|_{h^{*}}^{2} \\
= & \frac{4}{h^{2}}\left(\left\|D_{I^{-}}^{m+\theta} \delta_{x}^{-} U_{R}^{m+\theta}\right\|_{h^{*}}^{2}+\left\|D_{R^{-}}^{m+\theta} \delta_{x}^{-} U_{I}^{m+\theta}\right\|_{h^{*}}^{2}\right) \\
& +\frac{8}{h} \sum_{j=1}^{N} D_{R j^{-}}^{m+\theta} \delta_{x}^{-} U_{R j}^{m+\theta} D_{I j^{-}}^{m+\theta} \delta_{x}^{-} U_{I j}^{m+\theta} .
\end{aligned}
$$

From (2.25), (2.27) and (2.29),

$$
\begin{aligned}
\left\|\frac{U^{m+1}-U^{m}}{\Delta t^{m}}\right\|_{h}^{2} & \leq \frac{4}{h^{2}}\left(\left\|D_{R^{-}}^{m+\theta} \delta_{x}^{-} U^{m+\theta}\right\|_{h^{*}}^{2}+\left\|D_{I^{-}}^{m+\theta} \delta_{x}^{-} U^{m+\theta}\right\|_{h^{*}}^{2}\right) \\
& =\frac{4}{h^{2}}\left(\left(\left(D_{R^{-}}^{m+\theta}\right)^{2}+\left(D_{I^{-}}^{m+\theta}\right)^{2}\right) \delta_{x}^{-} U^{m+\theta}, \delta_{x}^{-} U^{m+\theta}\right)_{h^{*}} .
\end{aligned}
$$

Using assumption (2.12), we obtain

$$
\left\|\frac{U^{m+1}-U^{m}}{\Delta t^{m}}\right\|_{h}^{2} \leq \frac{4}{h^{2}} \max _{x_{j} \in \bar{\Omega}_{h}} \frac{\left|D_{j}^{m+\theta}\right|^{2}}{D_{R j}^{m+\theta}}\left\|\left(D_{R^{-}}^{m+\theta}\right)^{1 / 2} \delta_{x}^{-} U^{m+\theta}\right\|_{h^{*}}^{2} .
$$

Then, (2.24) implies that

$$
\begin{aligned}
& \frac{\left\|U^{m+1}\right\|_{h}^{2}-\left\|U^{m}\right\|_{h}^{2}}{2 \Delta t^{m}} \\
& +\left(1-\frac{2 \Delta t^{m}(1-2 \theta) \max _{x_{j} \in \bar{\Omega}_{h}} \frac{\left|D_{j}^{m+\theta}\right|^{2}}{D_{R j}^{m+\theta}}}{h^{2}}\right)\left\|\left(D_{R^{-}}^{m+\theta}\right)^{1 / 2} \delta_{x}^{-} U^{m+\theta}\right\|_{h^{*}}^{2} \leq 0 .
\end{aligned}
$$

If we assume that (2.11) holds then

$$
\left\|U^{m+1}\right\|_{h}^{2} \leq\left\|U^{m}\right\|_{h}^{2}, \quad m=0, \ldots, M-1 .
$$

We conclude that, in the case $\theta \in\left[0, \frac{1}{2}\right.$ [, the scheme (2.13) is stable provided that the condition (2.11) holds.

REMARK 1. In the case of Dirichlet boundary conditions $(\beta=0)$, we obtain a similar stability result defining the following discrete $L^{2}$ inner product

$$
(U, V)_{h}=\sum_{j=1}^{N-1} h U_{j} \bar{V}_{j} .
$$


REMARK 2. If one considers the complex diffusion $D$ as in (1.3) with fixed $\kappa$, then the stability condition (2.11) is equivalent to

$$
\Delta t^{m} \leq \frac{\left(\min \left\{h_{1}, \ldots, h_{d}\right\}\right)^{2} \cos \vartheta}{2 d(1-2 \theta)}\left(1+\frac{\min _{x_{j} \in \bar{\Omega}_{h}}\left(U_{I j}^{m}\right)^{2}}{\kappa^{2} \vartheta^{2}}\right), m=1, \ldots, M-1,
$$

which is less restrictive than the conjecture for stability condition by Gilboa et al. [7] where the multiplicative term on the right was minored by 1. Moreover, in (2.35) the most restrictive condition for the time step is obtained in the first iteration (since $\left.\operatorname{Im}\left(U_{j}^{0}\right)=0\right)$, therefore suggesting an adaptive approach of recomputing the step in time in every iteration (see [1]).

3. Numerical results. Having in mind the application in image filtering for the presented nonlinear complex diffusion method, we will focus on the numerical illustration of stability in this section. One wants to illustrate under which conditions is the noise is eliminated or amplified.

We have implemented the explicit $(\theta=0)$ scheme $(2.9)$ in $1 \mathrm{D}$ and 2D to illustrate the stability condition in Theorem 2.1, considering homogeneous Dirichlet boundary conditions. We considered $\vartheta=\pi / 180, \kappa=10$ for the complex diffusion filter (1.3), which is commonly used to remove noise while preserving edges in image processing $[1$, $11]$.

We point out that the function defined by (1.3) satisfies both assumptions (2.3) and (2.12) necessary in the above study of stability.

3.1. Example in 1D. We considered a profile with jump and ramp edges, namely considering a initial condition of

$$
U(x, 0)= \begin{cases}255, & 10 \leq x \leq 15 \\ 125, & 15<x \leq 20 \wedge 30 \leq x \leq 40 \\ \frac{255(30-x)+125(x-20)}{10}, & 20<x<30 \\ 0, & \text { otherwise, }\end{cases}
$$

and discretization points $x_{j}=j, j=1,2, \ldots, 51$, again with $h=1$. We considered the profile affected by additive noise has presented in figures 3.1 and 3.2. The stability condition is satisfied if $\Delta t \leq 0.49992$, being this value determined by the first iteration as mentioned in remark 2 .

In figure 3.1, we present the results for three different time steps and computed the approximating at two different times $T=1$ and $T=2$. In figure 3.2 and 3.3 we present the first and second iterations, respectively, with time steps close to the stability condition. It is clear in the close up in figure 3.2 that the instability arises in areas with jump edges whenever the stability condition is not fulfilled, while the scheme is stable if the condition is satisfied. Moreover, the second iteration presented in figure 3.3 is much smoother than the first one. This is due to the fact that the most restrictive stability condition is obtained for the first iteration, as mentioned in remark 2.

3.2. Examples in 2D. We considered two examples. The first one consists of a synthetic image as presented in figure 3.4, affected by additive noise. Reconstructions using the explicit finite difference scheme are presented in figures 3.4 and 3.5, illustrating the stability condition $\Delta t \leq 0.24996$ given by Theorem 2.1 for this case. 

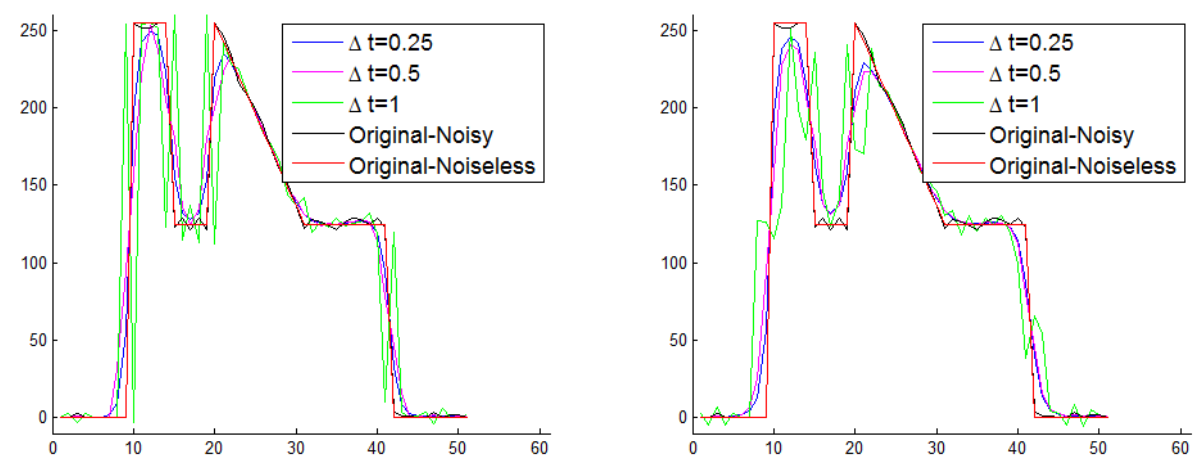

FIG. 3.1. Numerical solution for the explicit method with different time step and diffusion times of $T=1$ (left) and $T=2$ (right).
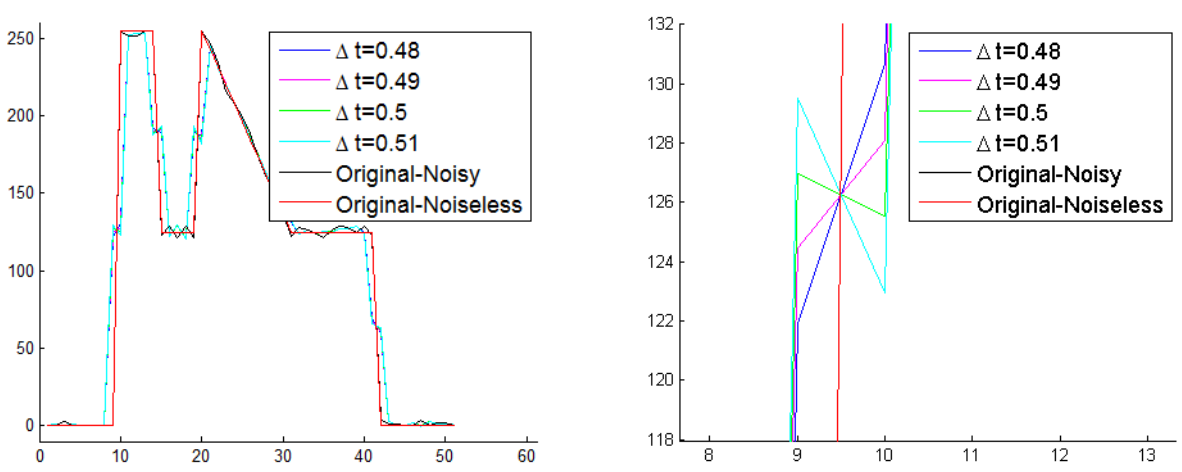

FIG. 3.2. First iteration (left) and close up in a edge (right) for the explicit method with time steps close to the cut-off value for stability $\Delta t=0.4992$.

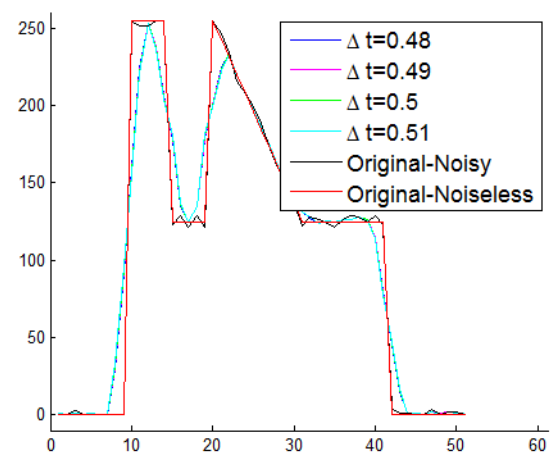

FIG. 3.3. Second iteration for the explicit method with time steps close to the cut-off value for stability $\Delta t=0.4992$. 

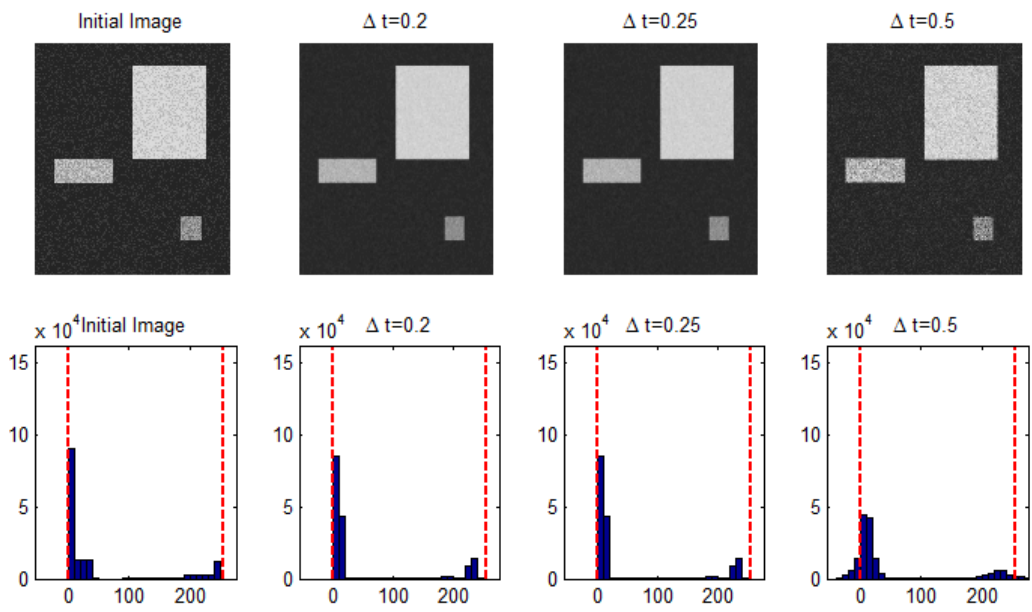

FIG. 3.4. Original and denoised images with the explicit method (top) and their histograms of intensities (bottom) with different time steps for a diffusion time of $T=1$.
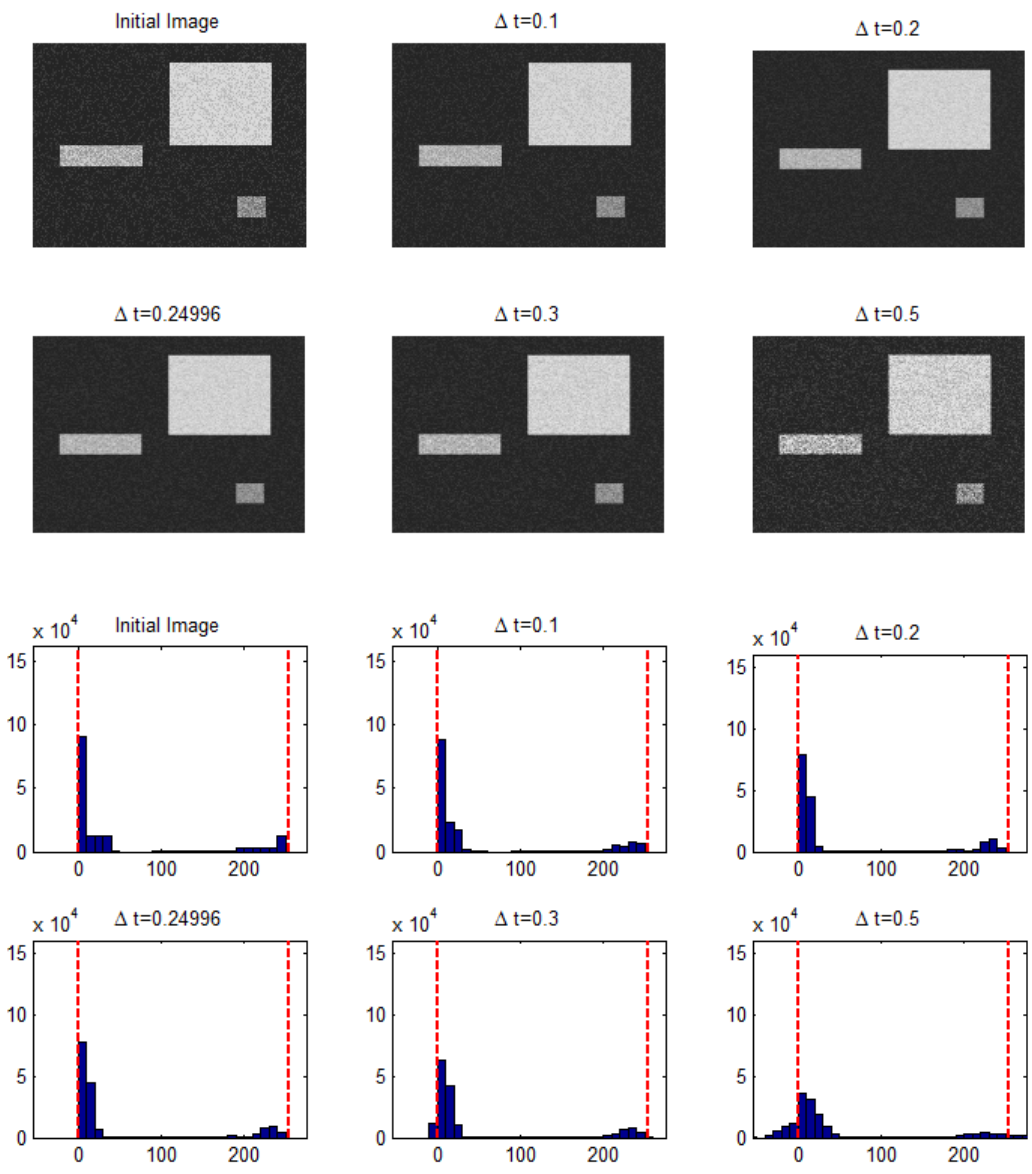

FIG. 3.5. Original and first iteration for the explicit method (top) and histograms of intensities (bottom) with time steps close to the cut-off value for stability $\Delta t=0.24996$. 

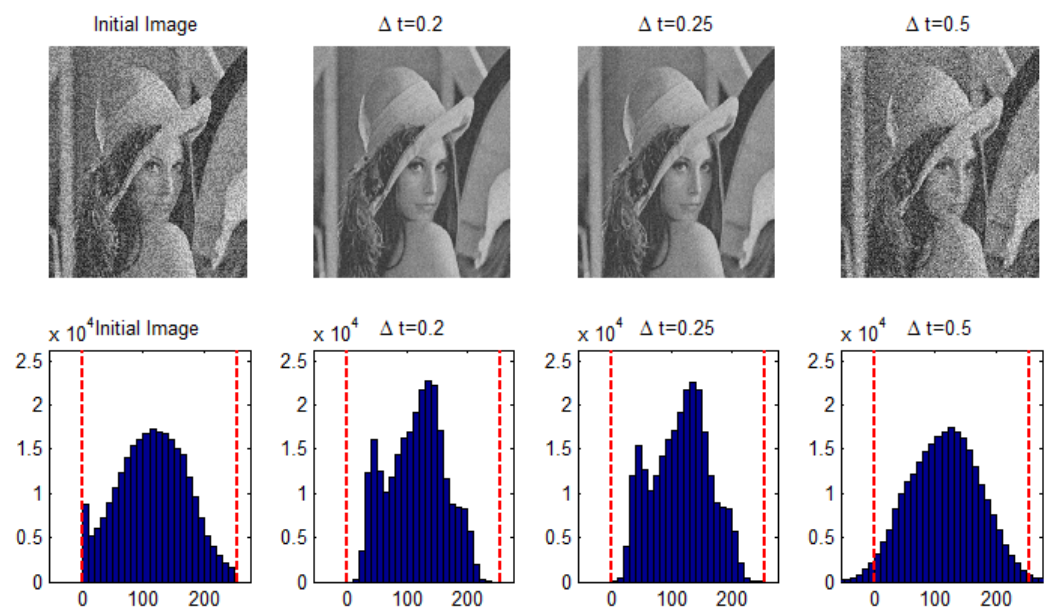

FIG. 3.6. Original and denoised images with the explicit method (top) and their histograms of intensities(bottom) with different time steps for a diffusion time of $T=1$.

The figures illustrate the stability condition. In fact, if the time step is greater than the cut-off (e.g. $\Delta t \geq 0.3$ ) the image presents intensities outside the image range $[0,255]$. Moreover, in this cases the noise is not eliminated but on the other hand seems to be amplified. For time steps slightly over to the stability condition (e.g. $\Delta t=0.25)$ the method seems to be stable, however the denoising effect seems to be less effective as for $\Delta t=0.2$.

We also considered a digital image example: Lena's image, commonly used in the image processing community. Figures 3.6 and 3.7 show the results in similar conditions to the previous example. The conclusions are also quite similar. The intensity values fall of the image range $[0,255]$ for $\Delta t \geq 0.3$ at the first iteration (see figure 3.7) and for a fixed diffusion time of $T=1$ it visually seems that the denoised image for $\Delta t=0.25$ (which is slightly over the stability condition) is not as smooth as for a smaller time step further away from the stability cut-off (see figure 3.6). This is clear in the hat's feather region of the image in figure 3.6).

4. Conclusion and Future work. In this paper we present a stability result for nonlinear complex diffusion. The stability condition concerns a class of finite difference schemes, that range from explicit $(\theta=0)$ to implicit $(\theta=1)$ through the well known Crank-Nicolson scheme $(\theta=1 / 2)$. Moreover, we illustrate the theoretical result with numerical examples both in $1 \mathrm{D}$ and $2 \mathrm{D}$. The $2 \mathrm{D}$ case is more relevant since nonlinear complex diffusion is commonly used for image denoising.

Two more remarks should be made in what concerns the numerical application of this scheme to image denoising. The first is that the time step used should not be close to the cut-off value for stability, since the denoising effect is not so effective as shown by the examples. The second remark is that it seems also profitable to use an adaptative time step along iterations, as already implicitly mentioned in Remark 2. The cut-off value for stability is smaller at early iterations and may allow the numerical scheme to take higher steps in time as iterations go by. In this way, the time step should be computed at each iteration having in mind the stability condition in Theorem 2.1, in the spirit of [1].

Future work might include the proof of convergence for finite difference schemes 

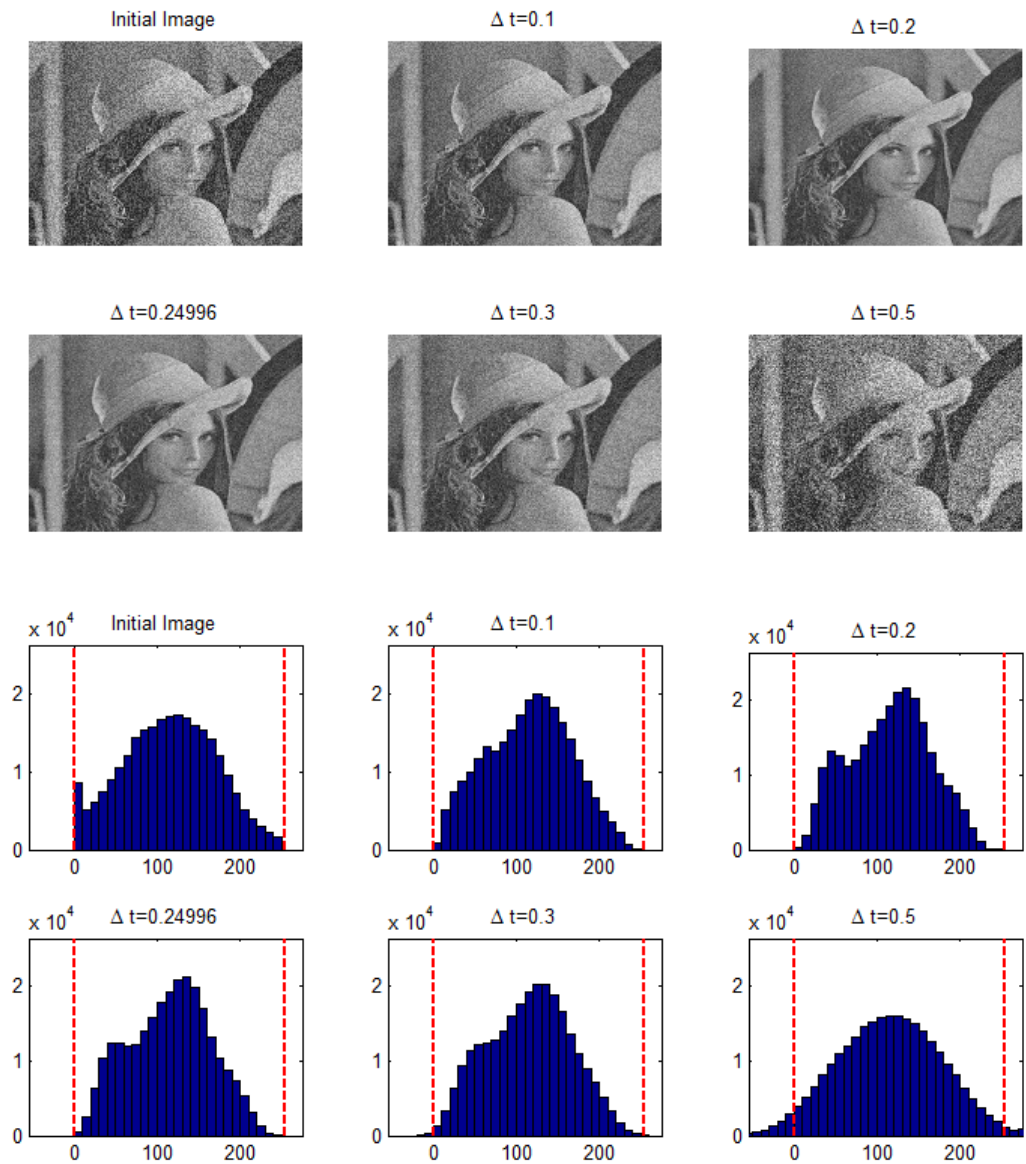

FIG. 3.7. Original and first iteration for the explicit method (top) and histograms of intensities (bottom) with time steps close to the cut-off value for stability $\Delta t=0.24996$.

for nonlinear complex diffusion. Lax equivalence theorem [9] for stability and convergence of consistent methods only holds in the linear case, therefore a different approach must be taken into account.

\section{REFERENCES}

[1] R. Bernardes, C. Maduro, P. Serranho, A. Araújo, S. Barbeiro, and J. Cunha-Vaz. Improved adaptive complex diffusion despeckling filter. Optics Express, 18 (23):24048-24059, 2010.

[2] T. Brox, A. Bruhn, N. Papenberg, and J. Weickert. Computer Vision - ECCV 2004. Lecture Notes in Computer Science, volume 3024, chapter High accuracy optical flow estimation based on a theory for warping, pages 25-36. Springer, Berlin, 2004.

[3] T. Chan and L. Shen. Stability analysis of difference schemes for variable coefficient Schrödinger type equation. SIAM J. Numer. Anal., 24 (2):336-349, 1987.

[4] J. Crank. The Mathematics of Diffusion. Clarendon Press, Oxford, second edition, 1975.

[5] L. C. Evans. Partial Differential Equations. Graduate Studies in Mathematics, 19. American Mathematics Society, Providence, RI, second edition, 2010.

[6] D. Fernández, H. Salinas, and C. Puliafito. Automated detection of retinal layer structures on optical coherence tomography images. Optics Express, 13 (25):10200-10216, 2005.

[7] G. Gilboa, N. Sochen, and Y. Zeeni. Image enhancement and denoising by complex diffusion 
processes. IEEE Trans Pattern Anal Mach Intell, 26 (8):1020-1036, 2004.

[8] H. Grossauer and O. Scherzer. Scale Space Methods in Computer Vision, Lecture Notes in Computer Science, volume 2695, chapter Using the Complex Ginzburg-Landau Equation for Digital Inpainting in 2D and 3D, pages 225-236. Springer, 2003.

[9] P. D. Lax and R. D. Richtmyer. Survey of the stability of linear finite difference equations. Comm. Pure Appl. Math., 9:267-29, 1956.

[10] P. Perona and J. Malik. Scale-space and edge detection using anisotropic diffusion. IEEE Trans Pattern Anal Mach Intell, 12 (7):629-639, 1990.

[11] H. Salinas and D. Fernández. Comparison of pde-based nonlinear diffusion approaches for image enhancement and denoising in optical coherence tomography. IEEE Trans. Med. Imaging, 26 (6):761-771, 2007.

[12] E. Süli. Finite Element Methods for Partial Differential Equations, Lecture notes. University of Oxford, 2005.

[13] J. W. Thomas. Numerical Partial Differential Equations: Finite Difference Methods. Texts in Applied Mathematics, 22. Springer, New York, 1995.

[14] J. Weickert. Anisotropic diffusion filters for image processing based quality control. Proc. 7th Eur. Conf. Mathematics in Industry, 1252:355-362, 1994.

[15] J. Weickert. A review of nonlinear diffusion filtering. In B. M. ter Haar Romeny, L. Florack, J. J. Koenderink, and M. A. Viergever, editors, Scale-Space, volume 1252 of Lecture Notes in Computer Science, pages 3-28. Springer, 1997.

[16] H. Zimmer, A. Bruhn, L. Valgaerts, M. Breuß, J. Weickert, B. Rosenhahn, and H.-P. Seidel. Vision, Modeling, and Visualization, chapter PDE-based anisotropic disparity-driven stereo vision, pages 263-272. AKA Heidelberg, 2008. 